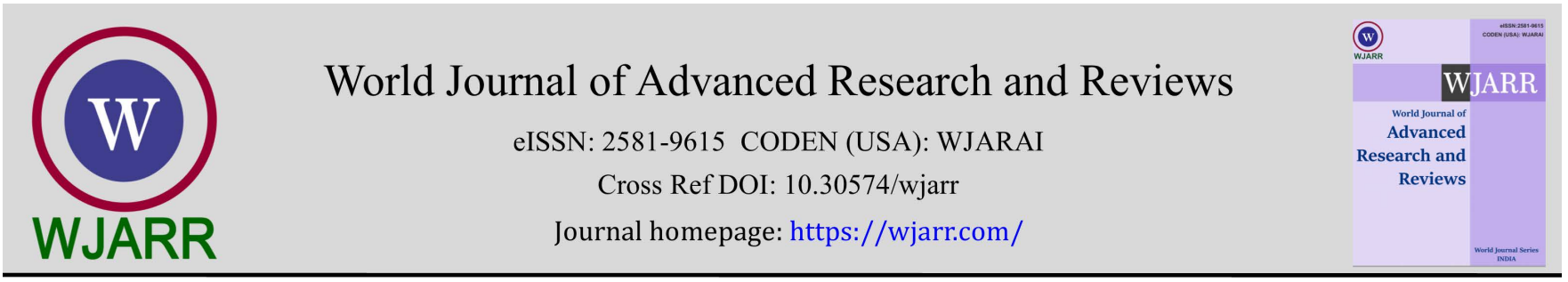

(CASE REPORT)

Check for updates

\title{
Trigeminal Ganglioneuroma: A rare case of trigeminal neuralgia caused by Meckel's cavum tumor: a case report and review of the literature
}

Brahim El Mostarchid *, Mamoune El Mostarchid, Allaoui Mohamed, Oukabli Mohamed, Mohamed Mouhssani, Asri Abad Chrif and Gazzaz Miloudi

Neurosurgery department of Mohamed V Military Teaching Hospital. Rabat. Mohamed V Souissi University. Rabat. Morocco.

World Journal of Advanced Research and Reviews, 2022, 13(02), 066-070

Publication history: Received on 25 December 2021; revised on 27 January 2022; accepted on 29 January 2022

Article DOI: https://doi.org/10.30574/wjarr.2022.13.2.0099

\begin{abstract}
Background: Ganglioneuroma (GN) is a rare benign tumor originating from the sympathetic nerves, and its origination from the trigeminal nerves is even rarer. Only six cases of GN originating from the trigeminal nerve have previously been reported.
\end{abstract}

Aim: To report a very rare case of GN arising in Mikel's cave revealed by atypical trigeminal neuralgia.

Case report: A 24-Year-old Moroccan man presented left trigeminal neuralgia. Intracranial MRI showed a 4.4 x $2.5 \times 3$ $\mathrm{cm}$ well-circumscribed multilobular mass in the posterior middle fossa and the petroclival area with compression to the medial temporal lobe. The tumor appeared homogeneously hypointense on T1WI, and hyperintense on T2WI and T2WI-Flair, and showed heterogeneous moderate enhancement. Bone window petrous CT scan showed thinning and remodeling bone on the left petrous apex. The preoperative diagnosis was cystic schwannoma. The patient underwent a subtemporal extradural approach with total removal of the lesion. The histological and immunohistochemically study confirmed the diagnosis of GN. Postoperative was marked by left keratitis for two months. No recurrence was documented on 3 years follow-up.

Conclusion: we described a benign and extremely rare trigeminal ganglioneuroma. This case is the seven-reported case in the literature. The subtemporal interdural approach must be considered in the surgical approach of trigeminal Meckel's GN. Trigeminal GN should be considered in the differential diagnosis of the tumor found in Mickel's cavum.

Keywords: Trigeminal neuralgia; Magnetic resonance imaging; Meckel's cavum; Middle-posterior cranial fossa surgery; Subtemporal extradural approach; Trigeminal nerve ganglioneuroma

\section{Introduction}

Ganglioneuromas (GN) are benign tumors of neural crest origin, which arise from the peripheral nervous system or adrenal glands. Intracranial involvement of GN is extremely rare and there have been only six cases reported of GN arising from the trigeminal nerve until 2017 [1-7].

We report a very rare case of trigeminal GN in the posterior middle fossa at Meckel's cavum area, revealed by atypical trigeminal neuralgia in a 24-year-old Moroccan man. Our case is the seven cases in the literature and the first Moroccan case trigeminal GN.

\footnotetext{
${ }^{*}$ Corresponding author: Brahim El Mostarchid

Neurosurgery department of Mohamed V Military Teaching Hospital. Rabat. Mohamed V Souissi University. Rabat. Morocco. 


\section{Case report}

A Moroccan military 24-year-old man immunocompetent was presented to our department with a one-year history of left atypical trigeminal neuralgia with episodic red eye. Neurological and laboratory investigations showed normal results. No visual disturbances were noted. Only hypoesthesia in the left V2 trigeminal face area was noted. All the neurologic examination was negative. The somatic examination was unremarkable.

A cranial CT scan showed a lesion situated in the left petrous bone with the bone remodeling of Meckel's cavum. The tumor deformed the petrous apex area. Bone window image showed thinning and remodeling bone on the petrous apex. (Figure 1: A.B).

Brain MRI revealed a $4 \times 2.5 \times 3 \mathrm{~cm}$ well-circumscribed multilobular mass in the posterior middle fossa and the petroclival area with mild compression to the medial temporal lobe. The tumor appeared homogeneously hypointense on T1WI, and hyperintense on T2WI and T2WI-Flair (Figure1: C.D), and showed heterogeneous moderate enhancement on contrast-enhanced imaging. Cerebral angiographic showed no tumoral blush.

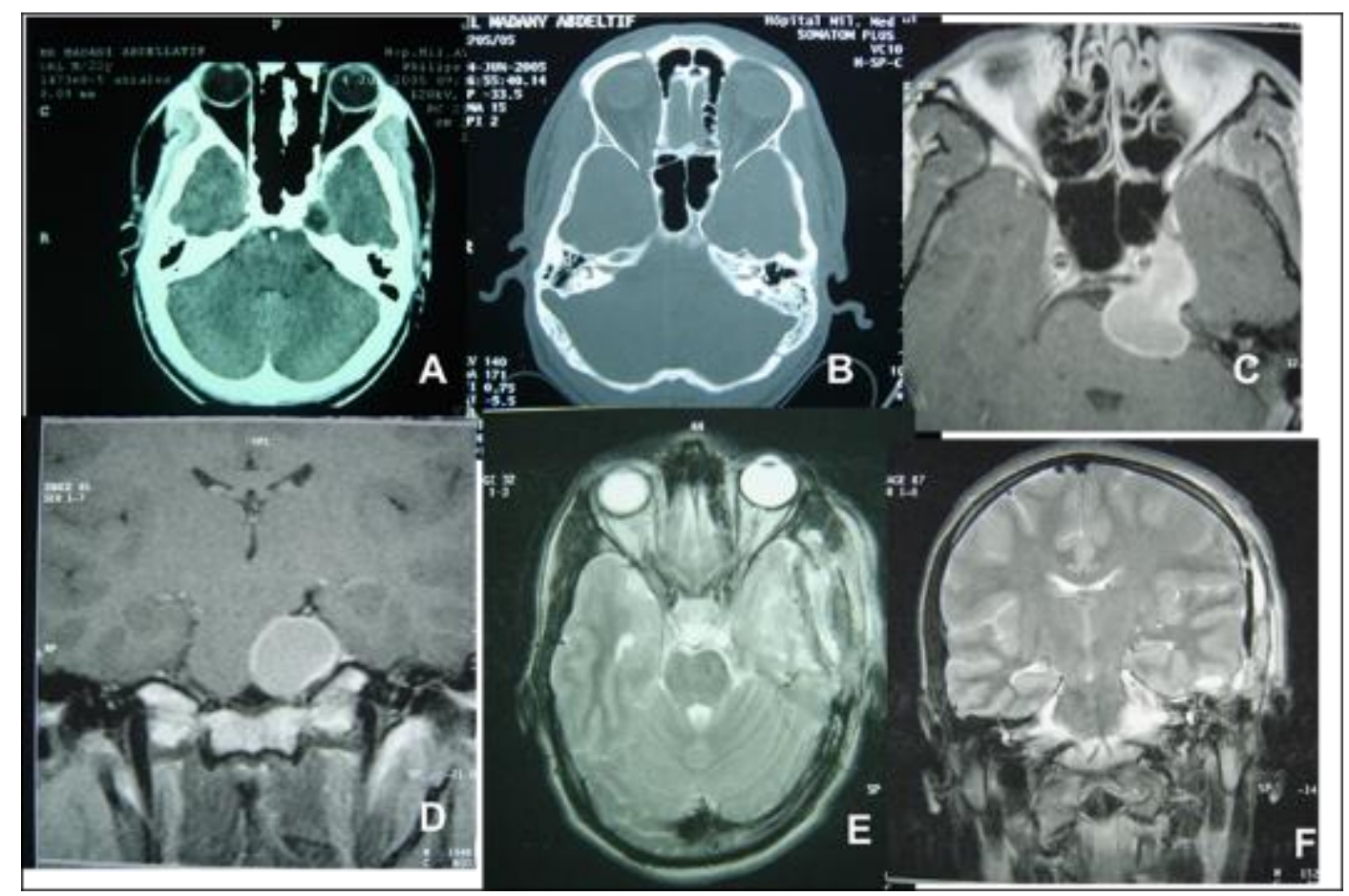

Figure 1 (A) Brain CT scan on axial view and bone window on axial view showing low homogeneous low-density mass in left apex petrous. (B). Bone window image showed thinning of the apex petrous bone. (C, D) MRI on T1-WI; on axial and coronal view: showing $4 \times 2.5 \times 3 \mathrm{~cm}$ well-circumscribed multilobular mass in the posterior middle fossa and petroclival area with mild compression to the medial temporal lobe, enhancement on contrast-enhanced imaging. Trigeminal ganglioneuroma. (E, F). Postoperative T2-WI MRI showing total removal of the lesion

Based on the clinical history, location, CT, and MR findings, the preoperative diagnosis was cystic trigeminal neuroma. The patient underwent a subsequent tumor resection through a subtemporal extradural approach. After subtemporal craniotomy, the temporal dura was raised from the skull base and dissected from the ophthalmic (V1), the maxillary (V2), mandibular division (V3), and Meckel's cave. A cystic component was evacuated first. A second fleshy component was removal in a piece-meal microsurgical manner. V2, V3 nerves were intact. The remodeling apex petrous bone was seen. Immediate postoperative was unremarkable with regression of trigeminal neuralgia. By left late keratitis at 10 days postoperatively, in the left eye. Treated by an ophthalmologist for three months by local therapy. Postoperative Follow-up MRI two months after operation showed a complete resection, with no residue enhancement detected (Fig.1. E). Histopathologic examination demonstrated a benign tumor composed of mature ganglion cells and spindle-shaped cells in an abundant collagenous stroma (Fig 2: A.B). Tumor cells were positive for S-100 protein by immunohistochemical staining. There was no immunoreactivity to anti-GFPA, antibody anticytokeratine (17, cytokeratin 7). and the pathologic diagnosis was ganglioneuroma. With 4 years of follow-up, this military man is in good health. No recurrence was documented. 


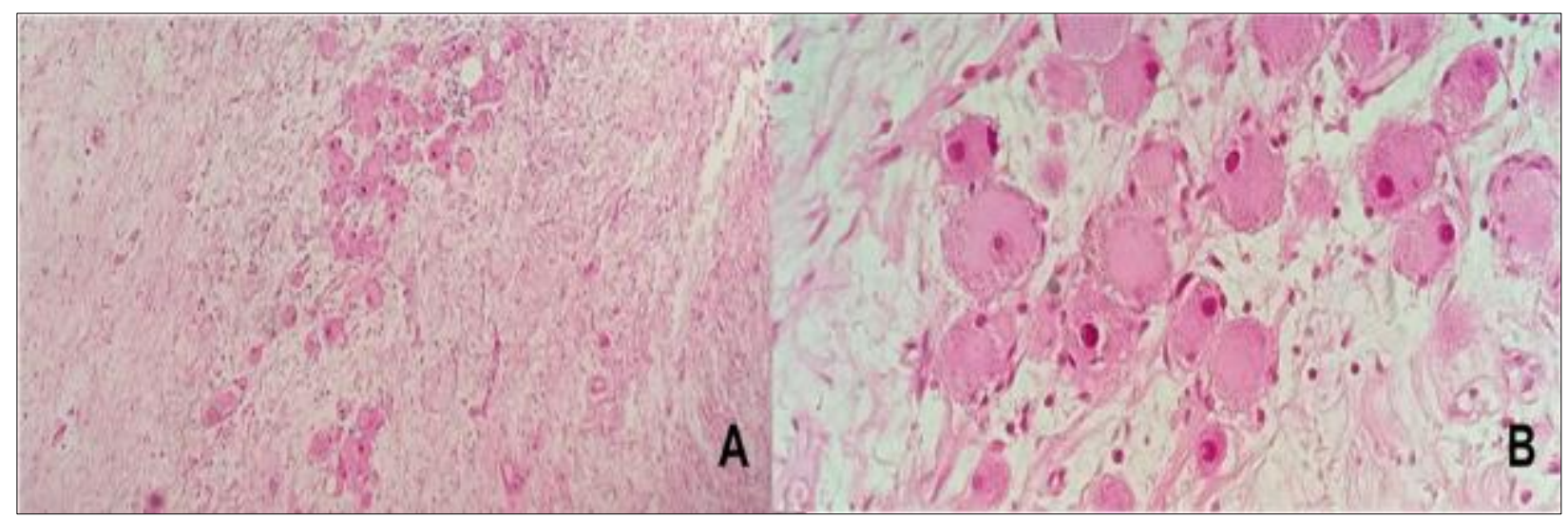

Figure 2 Histopathological examination shows that the tumor consists of mature ganglion cells and spindle-shaped cells with scanty myxoid changes, HE stains, x 10 (A). Photomicrograph shows mature ganglion cells HE stains x 40. (B). It's was a relatively strong S-100 expression of ganglion cells. Trigeminal ganglioneuroma in 25-year-old man

\section{Discussion}

Our patient presented atypical trigeminal neuralgia revealing a trigeminal ganglioneuroma. This finding is very rare. A review of literature can find only a few intracranial GN and only six cases involving trigeminal nerve [1-7].

GN is considered the most mature and noninvasive form of neuroblastic tumors. It derives from neural crest cells and can arise from wherever sympathetic tissue exists. The two most common locations for this tumor are retroperitoneum and posterior mediastinum; infrequently it occurs in the intracranial region [7].

Intracranial GN is very rare and tumors originating from the cerebellum, orbit, internal auditory canal, middle ear, sella turcica, cranial vault, and trigeminal nerve have been reported [9-15].

The case we report here is the seven descriptions of GN originating from the trigeminal nerve. 7). Only six cases of GN have been reported arising from the trigeminal nerve [1-7].

Histologically, these tumors are composed of single or clustered mature ganglion cells and mature Schwann cells. Ganglion cell tumors in the central nervous system (CNS) most frequently affect children and young adults, with about $80 \%$ of cases in most series occurring in patients under 30 years old $[3,5,7]$.

The typical sites of origin are the posterior middle cranial fossa, Meckel's cavum, and cerebellopontine angle consistent with the tracking of the trigeminal nerve. The largest diameter of the tumor ranged from 2.0 to $4.5 \mathrm{~cm}(\mathrm{mean}, 3.5 \mathrm{~cm})$.

Clinical presentation is variable. Our patient presented with atypical left trigeminal neuralgia. This finding is similar to the case of Khan N. [7] how reported a case of trigeminal neuralgia caused by cerebellopontine angle GN. Facial pain, periorbital pain, and numbness were reported by Kim SK [4]. The Abe T. [1] case was presented with seizures and upward gaze palsy. The Nakaguchi $\mathrm{H}$, case presented by sudden onset headaches [3]. Luo X [2] case was a radiological incidentaloma after motorbike trauma. Headaches and dizziness were the clinical findings in Deng X., case [5].

To the best of our knowledge, these are the seven cases of trigeminal GN; however, it is the first case reported in Morocco.

The MRI findings of GN have been elaborately described in the literature. Abe T and al. [1] reported the first case of trigeminal GN and described conventional MR imaging findings, which are similar to those of thoracoabdominal GN. The signal patterns of trigeminal GN are consistent with those of GN arising at other common locations [5].

These lesions are hypointense on T1-weighted MRI, heterogeneously hyperintense on T2 weighted images, and reveal mild to obvious heterogeneous enhancement. On DWI sequence, they are found to be a homogenously hyperintense signal with low ADC. Deng et al. [5] reported the tumor to show increased perfusion and both relative cerebral blood flow and volume on perfusion MRI. 
Kim SK. and al., [4] have reported a characteristic of Diffusion-weighted MRI findings and Magnetic resonance spectroscopy (MRS) in a patient with trigeminal GN. On MRS, the NAA peak is a specific marker of neuronal viability, and it may indicate the presence of neurons which are cell markers of a pathological diagnosis of GN, in extra-axial tumors. On single-voxel proton MRS, the tumor region didn't show markedly elevated choline (Cho) but show slightly lower levels of $\mathrm{N}$-acetylaspartate (NAA), and Cho/creatine $(\mathrm{Cr})$, and $\mathrm{NAA} / \mathrm{Cr}$, and a slightly higher Cho/NAA ratio compare to adjacent cerebellum [4]. On DWI sequence, they are found to be a homogenously hyperintense signal with low ADC. Deng et al. [4] reported the tumor to show increased perfusion and both relative cerebral blood flow and volume on perfusion MRI.

Advanced MRI showed the following tumor characteristics: significantly increased perfusion on perfusion imaging; isointense on diffusion-weighted imaging, whorled appearance within the tumor, and no significant signs of damage to the white matter fiber tracts in the fractional anisotropy color map, and compare to the adjacent brain tissue, Choline didn't show markedly elevation, and $\mathrm{N}$-acetylaspartate peak showed a slight reduction on magnetic resonance spectroscopy [5].

Histopathologically, ganglioneuroma is composed of single or clustered mature, giant ganglion cells and Schwannian stroma. It usually has no components of neuroblasts, intermediate cells, or mitotic figures that indicate malignant differentiation $[3,4,7]$.

According to the international neuroblastoma pathology classification of neuroblastic tumors, GN has been divided into two subtypes: maturing and mature subtype. Typical GN is composed of mature ganglion cells and schwannian stroma; however, tumors with entirely maturated ganglion cells are rare (approximately 7\%) [6] Immunohistochemically, it is characterized by reactivity for S-100, vimentin, synaptophysin, and neuronal markers. In our case; tumor cells were positive for S-100 protein by immunohistochemical staining and no immunoreactivity was noted with anti GFPA, antibody anticytokeratine (17, cytokeratin 7). Ki-67 is also an immunomarker of cellular proliferation [6].

Surgical management: Many neurosurgical approaches were used in the management of trigeminal GN according to the localization, extension of GN in the middle posterior fossa or posterior fossa. Frontorbitozygomatic and anterior petrosal approach was used. Tumor resection through a preauricular subtemporal interdural approach was reported by Wang [6]. We used the subtemporal epidural approach in our case with success. The prognosis of surgical excision of the tumor is favorable. It is generally accepted that surgical resection alone is curative for GN.

\section{Conclusion}

We described a benign and extremely rare trigeminal ganglioneuroma, which was lack of specific imaging characteristic, and were difficult to diagnose before operation. The subtemporal interdural approach must be considered in the surgical approach of trigeminal Meckel's GN. Although trigeminal GN is a very rare tumor, it should be considered in the differential diagnosis of tumors found in the Mickel's area or the cerebellopontine angle cistern.

\section{Compliance with ethical standards}

\section{Disclosure of conflict of interest}

No conflict of interest.

\section{Statement of informed consent}

Informed consent was obtained from all individual participants included in the study.

\section{References}

[1] Abe T, Asano T, Manabe T, Matsura H, Furuta T, Taguchi K. Trigeminal ganglioneuroma. Brain Tumor Pathol. 1999; 16:49-53.

[2] Luo X, Hu X, Ye J. Primary intracranial trigeminal ganglioneuroma: a case report with review of the literature. J Pract Radiol. 2010; 26: 1719-22.

[3] Nakaguchi H, Murakami M, Matsuno A, Yamazaki K, Ishida Y. Ganglioneuroma originating from the trigeminal nerve in the middle cranial fossa. Case report. Neurol Med Chir (Tokyo). 2012; 52: 95-8. 
[4] Kim SK, Jeong MY, Kang HK, Yoon W. Diffusion-weighted magnetic resonance imaging findings in a patient with trigeminal ganglioneuroma. Korean J Radiol. 2013; 14: 118-21.

[5] Deng X, Fang J, Luo Q, Tong H, Zhang W. Advanced MRI manifestations of trigeminal ganglioneuroma: a case report and literature review. BMC Cancer. 2016; 16(1): 694.

[6] Wang T, Ma L, Lou X, Bu B. Trigeminal Ganglioneuroma in the Middle-posterior Cranial Fossa: a Case Report. Chin Med Sci J. 2017; 32(2): 123-8.

[7] Khan N, Michael A, Choucair A, Bit-Ivan E. Trigeminal Ganglioneuroma: A Rare Case of Trigeminal Neuralgia Caused by Cerebellopontine Angle Tumor. World Neurosurg. 2017; 99: 81.

[8] Lonergan GJ, Schwab CM, Suarez ES, et al. Neuroblastoma, ganglioneuroblastoma, and ganglioneuroma: radiologic-pathologic correlation. Radiographics. 2002; 22: 911-34.

[9] Cogan DG, Poppen JL, Hicks PH. Ganglioneuroma of chiasm and optic nerves. Arch Ophthalmol. 1961; 65: 481482 .

[10] Katz MC, Kier EL, Schechter MM. The radiology of gangliogliomas and ganglioneuromas of the central nervous system. Neuroradiology. 1972; 4: 69-73.

[11] Ozluoglu LN, Yilmaz I, Cagici CA, et al. Ganglioneuroma of the internal auditory canal: a case report. Audiol Neurootol. 2007; 12: 160-4.

[12] Bekelis K, Meiklejohn DA, Missios S, et al. Ganglioneuroma of the internal auditory canal presenting as a vestibular schwannoma. Skull Base Reports. 2011; 1: 89-94.

[13] Arseni C, Horvath L, Carp N, et al. Intracranial ganglioneuromas in children. Acta Neurochir (Wien). 1975; 32 : 279-86.

[14] Estefano J, Algaba J, Gorostiaga F, et al. Ganglioneuroma of the middle ear. A propos of a case. An Otorrinolaringol Ibero Am. 1992; 19: 5-12.

[15] Qamouss O, Zoubeir Y, Oukabli M, Chahdi H, Harket A, Abouchadi A, et al. [ganglioneuroma of the zygoma]. Rev Stomatol Chir Maxillofac. 2006; 107: 370-372. 